\title{
Selective serotonin reuptake inhibitors and venlafaxine in early pregnancy and risk of birth defects: population based cohort study and sibling design
}

\author{
Kari Furu, ${ }^{1}$ Helle Kieler, ${ }^{2}$ Bengt Haglund, ${ }^{2}$ Anders Engeland, ${ }^{13}$ Randi Selmer, ${ }^{1}$ Olof Stephansson, ${ }^{24}$ \\ Unnur Anna Valdimarsdottir, ${ }^{6} 6$ Helga Zoega, ${ }^{5}$ Miia Artama, ${ }^{78}$ Mika Gissler, ${ }^{910}$ Heli Malm, ${ }^{11} 12$ \\ Mette Nørgaard ${ }^{13}$
}

\section{ABSTRACT}

OBJECTIVE

To assess whether use of specific selective serotonin reuptake inhibitors (SSRIs) or venlafaxine in early pregnancy is associated with an increased risk of birth defects, with emphasis on cardiovascular birth defects even when accounting for lifestyle or other familial confounding.

DESIGN

Multicountry population based cohort study, including sibling controlled design.

SETTING
Nordic population (Denmark, Finland, Iceland, Norway,
and Sweden) identified from nationwide health
registers at different periods in 1996-2010.
POPULATION
The full study cohort included women giving birth to
2.3 million live singletons. The sibling cohort included
2288 singleton live births. The sibling controlled
analyses included sibling pairs who were discordant
for exposure to SSRIs or venlafaxine and birth defects.
MAIN OUTCOME MEASURE
Prevalence of birth defects, including subtypes of
cardiac defects. Odds ratio of birth defects from
logistic and conditional logistic regression.
RESULTS
Among 36772 infants exposed to any SSRI in early
pregnancy, 3.7\% ( $\mathrm{n}=1357$ ) had a birth defect
compared with $3.1 \%$ of 2266875 unexposed infants,
yielding a covariate adjusted odds ratio of 1.13 ( $95 \%$
confidence interval 1.06 to 1.20 ). In the sibling
controlled analysis the adjusted odds ratio decreased

\section{WHAT IS ALREADY KNOWN ON THIS TOPIC}

The teratogenicity of individual selective serotonin reuptake inhibitors (SSRIs) and venlafaxine remains controversial

Few studies have had sufficient power to study the direct effect of individual SSRIs on specific birth defects

\section{WHAT THIS STUDY ADDS}

In this large population based cohort study including sibling controlled analyses, there was no substantial increase in prevalence of overall cardiac birth defects among infants exposed to SSRIs or venlafaxine in utero

Although the prevalence of right ventricular outflow tract defects was higher in exposed infants in the full cohort, no association was found in the sibling controlled analyses

The general absence of strong associations between birth defects and SSRIs and venlafaxine is reassuring to 1.06 (0.91 to 1.24$)$. The odds ratios for any cardiac birth defect with use of any SSRI or venlafaxine were 1.15 (95\% confidence interval 1.05 to 1.26$)$ in the covariate adjusted analysis and 0.92 (0.72 to 1.17) in the sibling controlled analysis. For atrial and ventricular septal defects the covariate adjusted odds ratio was 1.17 (1.05 to 1.31). Exposure to any SSRI or venlafaxine increased the prevalence of right ventricular outflow tract obstruction defects, with a covariate adjusted odds ratio of 1.48 (1.15 to 1.89). In the sibling controlled analysis the adjusted odds ratio decreased to 0.56 ( 0.21 to 1.49) for any exposure to SSRIs or venlafaxine and right ventricular outflow tract obstruction defects.

\section{CONCLUSIONS}

In this large Nordic study no substantial increase was found in prevalence of overall cardiac birth defects among infants exposed to SSRIs or venlafaxine in utero. Although the prevalence of septal defects and right ventricular outflow tract defects was higher in exposed infants, the lack of an association in the sibling controlled analyses points against a teratogenic effect of these drugs.

\section{Introduction}

Depression is common in pregnancy. ${ }^{1}$ Selective serotonin reuptake inhibitors (SSRIs) are the most frequently used antidepressants in pregnant women, with as many as 3\% of such women in Europe and 4-10\% in North America receiving these drugs. ${ }^{23-6}$

The results of previous studies assessing the risk of birth defects after use of SSRIs in pregnancy have been conflicting. ${ }^{7}$ Most consistently reported were cardiovascular birth defects. ${ }^{8-15}$ One study ${ }^{16}$ found an increased risk of cardiovascular defects with combined use of SSRIs or venlafaxine and benzodiazepines. Increased risks of anal atresia, ${ }^{7}$ hypospadias, ${ }^{714}$ clubfoot, ${ }^{7}$ limb reduction defects, ${ }^{7}$ craniosynostosis, ${ }^{8}$ omphalocele, ${ }^{78}$ gastroschisis, ${ }^{8}$ and cystic kidneys ${ }^{17}$ have also been reported with use of SSRIs in early pregnancy, but less consistently. A few studies reported no increased risk of congenital birth defects associated with use of SSRIs in pregnancy. ${ }^{131819}$

Venlafaxine is a serotonin-norepinephrine reuptake inhibitor used increasingly as an alternative to SSRIs. ${ }^{20}$ Recently, a case-control study from the United States, based on 77 exposed cases and 14 exposed controls, reported that use of venlafaxine in early pregnancy was associated with several types of birth defects, with odds ratios ranging from 1.5 to $6.3 .{ }^{21}$ 
Limitations of previous studies include uncertain information on drug use, recall bias from self reports, limited information on confounding factors, and low statistical power. Larger studies are necessary for more definitive conclusions about particular types of birth defects and associations with specific drugs, rather than classes of drugs. ${ }^{22}$

The Nordic countries, representing a total population of about 26 million people, have similar nationwide registers of births and dispensed drugs, enabling large studies on the effects of rare drug exposures during pregnancy on birth defects. ${ }^{23}$ We performed a population based cohort study and a sibling controlled analysis within five Nordic countries to assess predefined and previously reported associations between exposure to individual SSRIs or venlafaxine in the first trimester and the risk of specific birth defects, with emphasis on cardiovascular defects, adjusting for important covariates such as familial factors.

\section{Methods}

\section{Data sources and study population}

In this population based study we included women from Denmark, Finland, Iceland, Norway, and Sweden who gave birth to a live singleton infant between 1996 and 2010. From nationwide health registers in each Nordic country we obtained data on births, dispensed drugs, birth defects, and potential confounders. These registers include prospectively collected health information on all inhabitants. A civil personal registration number is assigned to each resident at birth or immigration, enabling data linkage between the registers. Reporting to the registers is mandatory and regulated by national laws.

Each participating country contributed data for different periods corresponding to the availability of the prescription databases and other health registers. Accordingly we identified all live born singletons in Denmark 1997-2010, Finland 1996-2006, Iceland 200307, Norway 2005-10, and Sweden 2006-10. We also identified siblings born to the same mother during these periods.

\section{Ascertainment of exposure}

The Nordic prescription registers include data on dispensed drugs, substance, brand name, and formulation, together with date of dispensing. ${ }^{23}$ All drugs are classified according to the World Health Organization's Anatomical Therapeutic Chemical (ATC) classification. ${ }^{24}$ The following SSRIs were included in the analyses: fluoxetine (ATC code: N06AB03), citalopram (N06AB04), paroxetine (N06AB05), sertraline (N06AB06), fluvoxamine (N06AB08), and escitalopram (N06AB10), and in addition venlafaxine (N06AX16). We considered infants to have been exposed in utero if they were born to women who filled a prescription for an SSRI from 30 days before the first day of the last menstrual period until the end of the first trimester (defined as 97 days after the last menstrual period).

\section{Major birth defects}

From the medical birth, patient, and malformation registers we retrieved data on maternal characteristics, the pregnancy and delivery, and major birth defects diagnosed within 365 days after birth. The patient registers record information on diagnoses and hospital visits. ${ }^{2526}$ During the study period, birth defects were recorded according to the international classification of diseases, 10th revision (ICD-10) in Denmark, Norway, Sweden, and Iceland, whereas ICD-9-CM codes (Atlanta modification for birth defects) were used in Finland (see appendix 1 on bmj.com for ICD codes).

We categorised cardiovascular birth defects into any cardiac defects; situs anomalies and looping defects; conotruncal and major aortic arch anomalies; ventral septal defect or atrial septal defect; atrioventricular septal defects; right ventricular outflow tract obstruction defects; and left ventricular outflow tract obstruction defects. In addition we assessed several specific birth defects based on results from previous studies $^{891517}$ : anal atresia, hypospadias, clubfoot, limb reduction defects, craniosynostosis, omphalocele, gastroschisis, and cystic kidneys.

\section{Potential confounding factors}

We included the potential confounders of country of residence, maternal age at delivery, calendar year of delivery, birth order, maternal smoking during pregnancy, maternal diabetes, and concurrent drug use. The presence of maternal diabetes was defined as dispensed antidiabetic drugs (ATC code: A10) from 90 days before the first day of the last menstrual period to 97 days after the last menstrual period. Concurrent drugs considered as confounders included antiepileptics (N03), anxiolytics and hypnotics (N05B, N05C), and agents acting on the renin-angiotensin system (C09) dispensed from 30 days before the first day of the last menstrual period until 97 days after the last menstrual period.

\section{Statistical analyses}

We used logistic regression analysis to estimate odds ratios with 95\% confidence intervals for all major and specific types of birth defects according to exposure status, with adjustment for the included confounders. Owing to missing data on confounders, the number included in the adjusted analyses $(n=2145050)$ was slightly lower than the number included in the crude analyses $(n=2303647)$. In our primary analyses we assessed odds ratios for birth defects by exposure in early pregnancy to any SSRI and venlafaxine and to each specific SSRI or venlafaxine. In each instance the reference group comprised births of infants not exposed to any antidepressant (ATC code N06A) in utero. We present only the results from analyses with more than five births among exposed infants with the specific defect or defect group.

To adjust further for potential confounding from family related factors, we conducted sibling controlled analyses by restricting the study population to women with at least two children in the dataset. Furthermore, we restricted the sibling analyses to families with 


\begin{tabular}{|c|c|c|c|c|}
\hline \multirow[b]{2}{*}{ Characteristics } & \multicolumn{2}{|l|}{ No (\%) in full cohort } & \multicolumn{2}{|c|}{ No (\%) in sibling cohort } \\
\hline & Any SSRIt $(n=36772)$ & No SSRI $(n=2266875)$ & Any SSRIt $(n=980)$ & No SSRI $(n=1308)$ \\
\hline \multicolumn{5}{|c|}{ Maternal age at delivery (years): } \\
\hline$\leq 24$ & $6192(16.8)$ & 359817 (15.9) & $172(17.6)$ & $459(35.1)$ \\
\hline $25-34$ & $22209(60.4)$ & $1490718(65.8)$ & $643(65.6)$ & $732(56.0)$ \\
\hline $35-44$ & $8304(22.6)$ & $413939(18.3)$ & $165(16.8)$ & $117(8.9)$ \\
\hline$\geq 45$ & $67(0.2)$ & $2401(0.1)$ & $0(0.0)$ & $0(0.0)$ \\
\hline \multicolumn{5}{|l|}{ Birth order: } \\
\hline 1 & $16439(44.7)$ & $962629(42.5)$ & $217(22.1)$ & $481(36.8)$ \\
\hline $2-3$ & $17014(46.3)$ & $1134522(50.0)$ & $573(58.5)$ & $650(49.7)$ \\
\hline$\geq 4$ & $3113(8.5)$ & $151386(6.7)$ & $186(19.0)$ & $166(12.7)$ \\
\hline Missing & $206(0.6)$ & $18338(0.8)$ & $4(0.4)$ & $11(0.8)$ \\
\hline \multicolumn{5}{|c|}{ Maternal smoking in early pregnancy: } \\
\hline No & $24611(66.9)$ & $1810213(79.9)$ & $656(66.9)$ & $887(67.8)$ \\
\hline Yes & $9371(25.5)$ & $317437(14.0)$ & $288(29.4)$ & $373(28.5)$ \\
\hline Missing & $2790(7.6)$ & $139225(6.1)$ & $36(3.7)$ & $48(3.7)$ \\
\hline \multicolumn{5}{|c|}{ Maternal diabetesf: } \\
\hline Yes & $526(1.4)$ & $16992(0.7)$ & $18(1.8)$ & $11(0.8)$ \\
\hline No & $36246(98.6)$ & $2249883(99.3)$ & $962(98.2)$ & $1297(99.2)$ \\
\hline \multicolumn{5}{|c|}{ Exposed to other prescribed drugs§: } \\
\hline Yes & $5362(14.6)$ & $22315(1.0)$ & $140(14.3)$ & $55(4.2)$ \\
\hline No & $31410(85.4)$ & $2244560(99.0)$ & $840(85.7)$ & $1253(95.8)$ \\
\hline \multicolumn{5}{|c|}{$\begin{array}{l}\text { *The full study cohort included } 2303647 \text { singleton live births in five Nordic countries, 1996-2010, and the sibling cohort included } 2288 \text { singleton live births. } \\
\text { tDefined as dispensed SSRIs or venlafaxine to mothers at least once from } 30 \text { days before first day of last menstrual period to } 97 \text { days after last menstrual period. } \\
\text { fDefined as dispensed drugs used for diabetes (ATC code A10) to mothers at least once from } 90 \text { days before first day of last menstrual period to } 97 \text { days } \\
\text { after last menstrual period. } \\
\text { §Defined as the following drugs dispensed to mothers, antiepileptics (ATC code N03A), anxiolytics and hypnotics (N05B, N05C), angiotensin converting } \\
\text { enzyme inhibitors (CO9), at least once from } 30 \text { days before first day of last menstrual period to } 97 \text { days after last menstrual period. }\end{array}$} \\
\hline
\end{tabular}

siblings who were discordant for both exposure and outcome. Using conditional logistic regression we compared the prevalence of birth defects overall, any cardiac defects, and right ventricular outflow tract obstruction defects in infants exposed to any SSRI and venlafaxine with the prevalence in siblings not exposed to any antidepressants.

Predefined sensitivity analyses were performed for a shorter and a longer period of exposure-that is, dispensed drugs from the first day of the last menstrual period until 97 days after the last menstrual period and from 90 days before the first day of the last menstrual period until 97 days after the last menstrual period, respectively. In addition we conducted secondary multivariable analyses stratified by use of anxiolytics and hypnotics. Finally, to account for confounding by severity of underlying psychiatric condition, we restricted the primary analyses to pregnant women without a previous hospital admission for a psychiatric condition.

All analyses were conducted using SAS software, version 9.3 (SAS Institute, Cary, NC). The study was approved and performed according to ethical and legal requirements in each country. ${ }^{23}$

\section{Patient involvement}

There was no patient involvement in this study.

\section{Results}

The full study cohort included 2303647 singleton live births and the sibling cohort included 2288 singleton live births. In the full study cohort, 36772 (1.6\%) infants were exposed to a selective serotonin reuptake inhibitor
(SSRI) or venlafaxine during the first trimester in 19962010. The proportion of infants exposed to an SSRI or venlafaxine increased during the study period: from $0.6 \%$ in $1996-2000$ to $1.5 \%$ in $2001-05$ and $2.2 \%$ in $2006-10$. Table 1 presents the characteristics of the full study cohort and the sibling cohort according to drug exposure status. Citalopram and sertraline were the most commonly used SSRIs (table 2).

Among the 36772 infants exposed to SSRIs or venlafaxine during the first trimester, 1357 (3.7\%) had a diagnosis of a major birth defect compared with 71374 (3.2\%) of the 2266875 unexposed infants (adjusted odds ratio $1.13,95 \%$ confidence interval 1.06 to 1.20 , fig 1 ).

\begin{tabular}{|c|c|}
\hline Substance (ATC code) & No (\%) of infants \\
\hline Any SSRI* (N06AB or N06AX16) & $36772(1.60)$ \\
\hline Fluoxetine (N06AB03) & $6250(0.27)$ \\
\hline Citalopram (N06AB04) & $11193(0.48)$ \\
\hline Paroxetine (N06AB05) & $2879(0.12)$ \\
\hline Sertraline (N06AB06) & $7245(0.31)$ \\
\hline Fluvoxamine (N06AB08) & $255(0.01)$ \\
\hline Escitalopram (N06AB10) & $3950(0.17)$ \\
\hline Venlafaxine (N06AX16) & $2763(0.12)$ \\
\hline Mixedt & $2237(0.10)$ \\
\hline Other antidepressants $\ddagger$ & $7188(0.31)$ \\
\hline \multicolumn{2}{|c|}{$\begin{array}{l}\text { ATC=Anatomical Therapeutic Chemical classification. }{ }^{24} \\
{ }^{*} \text { Defined as dispensed selective serotonin-reuptake inhibitors (ATC } \\
\text { N06AB) or venlafaxine (N06AX16) at least once from } 30 \text { days before first } \\
\text { day of last menstrual period to } 97 \text { days after last menstrual period. } \\
\text { tExposed to more than one of the listed drugs. } \\
\text { †Exposed to any other antidepressant drug (ATC N06A) not listed. These } \\
\text { are excluded from analyses. }\end{array}$} \\
\hline
\end{tabular}




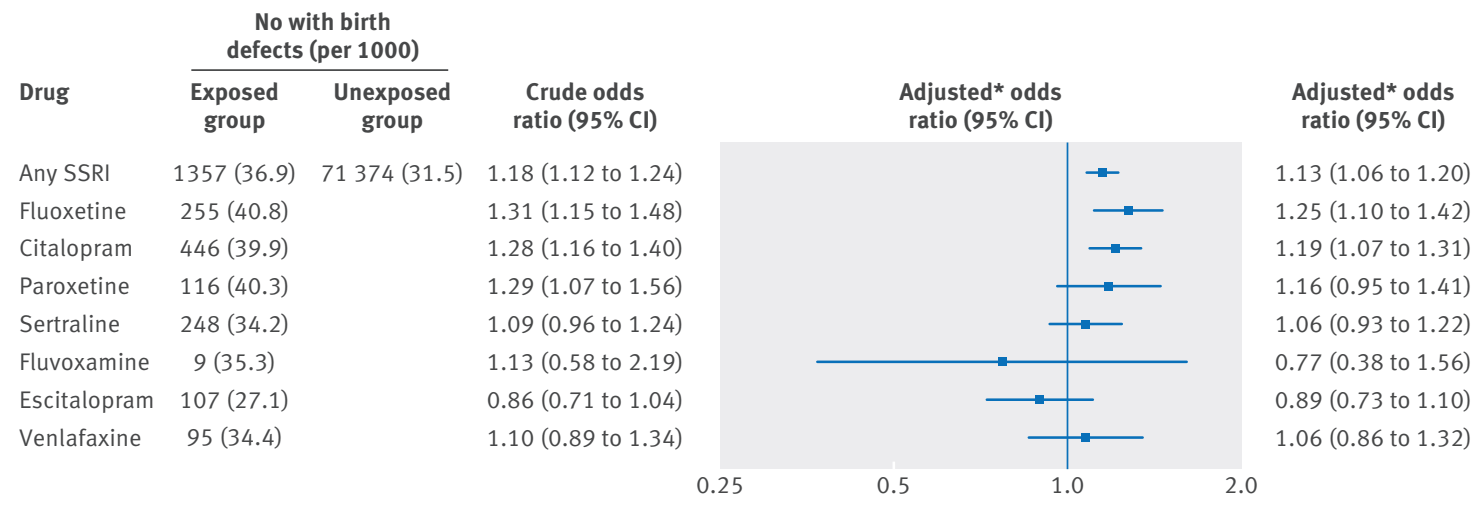

Fig 1 | Selective serotonin reuptake inhibitors (SSRIs) or venlafaxine in early pregnancy and risk of birth defects. *Adjusted for maternal age, year of birth, birth order, smoking, maternal diabetes, country, and use of other prescribed drugs (antiepileptics (ATC code N03), anxiolytics and hypnotics (N05B and N05C), and angiotensin converting enzyme inhibitors (C09))

The prevalence of overall cardiac birth defects was $1.5 \%$ among infants exposed to any SSRI or venlafaxine compared with $1.2 \%$ among infants in the unexposed group $(1.15,1.05$ to 1.26$)$. Apart from escitalopram, in utero exposure to each specific SSRI and to venlafaxine was associated with an increased prevalence of cardiac birth defects, with adjusted odds ratios between 1.13 and 1.34 . Similarly, and except for escitalopram, atrial or ventricular septal defects were positively associated with maternal use of each specific SSRI and with venlafaxine (fig 2).

The adjusted odds ratio for right ventricular outflow tract obstruction defects after exposure to any SSRI was 1.48 (1.15 to 1.89), with estimates for specific SSRIs varying from 1.40 to 2.54 (fig 2). Use of paroxetine was associated with an increased prevalence of conotruncal and major arch anomalies (2.27, 1.01 to 5.07), whereas associations with other SSRIs were less clear.

The prevalence of clubfoot and omphalocele was increased after exposure to any SSRIs, but we found no increased prevalence of hypospadias, limb reduction, craniosynostosis, gastroschisis, or cystic kidneys (fig 3). Exposure to citalopram and sertraline were both associated with an increased prevalence of clubfoot, and sertraline also with anal atresia.

In the sibling controlled analyses we identified 895 families with at least one exposed pregnancy and at least one birth with a malformation (tables 1 and 3). The adjusted odds ratios in the sibling controlled analyses were attenuated compared with estimates from the covariable adjusted analyses in the full cohort. The confidence intervals were also wider in the sibling controlled analyses.

The level of the odds ratios was not influenced by the order of exposure and outcome between the discordant siblings-that is, a first exposed sibling with a birth defect and an unexposed second sibling without a birth defect would yield odds ratios similar to a first unexposed sibling with no birth defects and a second exposed sibling with a birth defect.

In the predefined sensitivity analyses, using two different time windows for exposure, the effect estimates for birth defects associated with any SSRI were similar to those in the primary analyses. Stratification according to use of anxiolytics and hypnotics did not result in substantial variation of risk estimates between strata. The estimates were not substantially changed by restricting the primary analyses to women without previous admissions to hospital for psychiatric disorders (data not shown).

\section{Discussion}

In this large multinational population based study including over 2.3 million births from the Nordic countries, exposure to selective serotonin reuptake inhibitors (SSRIs) in utero was associated with a $13 \%$ increased prevalence of major birth defects and a 15\% increased prevalence of cardiac defects in a covariate controlled analysis. However, we found no increased prevalence in the sibling controlled analyses, suggesting that the small observed increased risk when including mothers and their infants in the analyses could be explained by familial related factors or other lifestyle related factors not adjusted for. Similarly, each specific SSRI increased the prevalence of right ventricular outflow tract obstruction defects, with adjusted odds ratios ranging from 1.40 to 2.54, whereas no association was found in the sibling controlled analysis ( $0.71,0.28$ to 1.78). No significant association was found between venlafaxine and birth defects.

\section{Comparisons with other studies}

Some of the births in our study have been included in previous national studies. ${ }^{11} 121527$ The observed increased prevalence of overall major birth defects in association with exposure to SSRIs was lower than the $21-30 \%$ increased risk reported in previous Danish studies, ${ }^{11} 1227$ but extends the findings from a previous Finnish study reporting no substantial increased risk of overall birth defects. ${ }^{15}$ In our data, SSRI use was associated with an increased prevalence of cardiovascular defects; mainly driven by a 30\% increase after exposure to paroxetine or fluoxetine, which is an effect of similar size to that reported from databases in the United Kingdom ${ }^{18}$ and the United States. ${ }^{8}$ Previous studies reporting risks of birth defects in association with the combined use of SSRIs and benzodiazepines included 


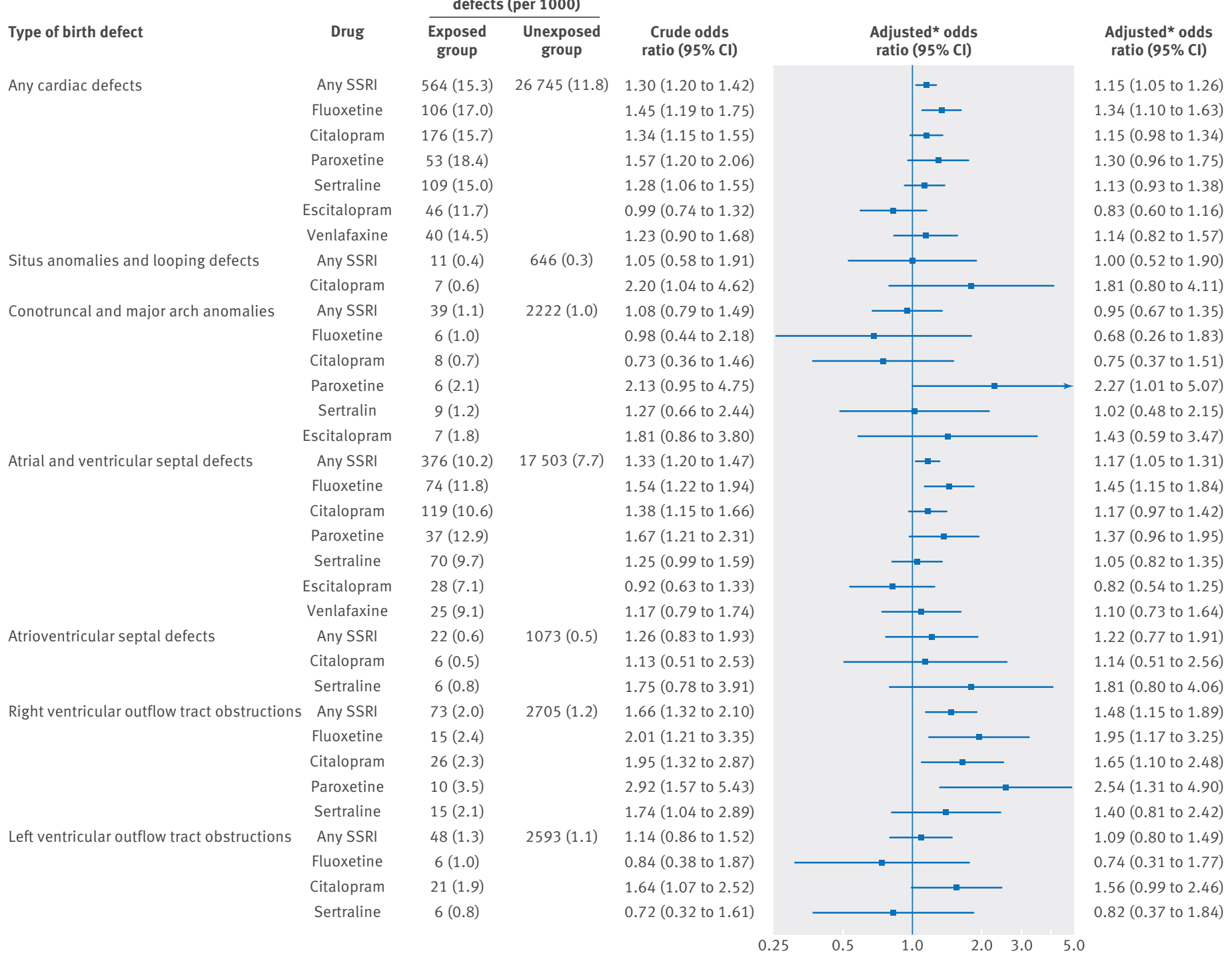

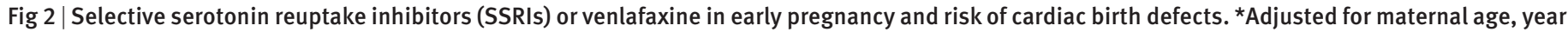

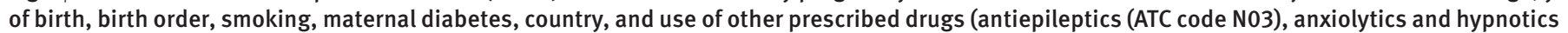
(N05B and N05C), and angiotensin converting enzyme inhibitors (C09)) small numbers of exposed infants, with ambiguous findings. ${ }^{1628}$ Our study showed no increased risk of birth defects with combined use of these drugs.

Our estimated $45 \%$ increased prevalence of septal defects associated with exposure to fluoxetine is an extension of the previous findings from Denmark ${ }^{1112}$ and Finland. ${ }^{15}$ In contrast, one study ${ }^{8}$ reported no association between maternal use of fluoxetine and septal defects (adjusted odds ratio 1.0, 95\% confidence interval 0.5 to 2.2), but given the lack of precision, those estimates may well have been in line with our findings. In our data, exposure to paroxetine was associated with a nearly $40 \%$ increased prevalence of septal defects, which contrasts with previous findings from Denmark, ${ }^{11} 12$ Finland, ${ }^{15}$ and the USA, ${ }^{8}$ but is in accordance with findings from the Netherlands (adjusted odds ratio 1.6, 95\% confidence interval 0.4 to 5.6). ${ }^{29}$ However, the estimated effect of sertraline on septal defects in our data does not support previous findings from Denmark ${ }^{112}$ and the
$\mathrm{USA},{ }^{8}$ reporting a twofold to threefold increased prevalence of septal defects in offspring exposed to sertraline. We found exposure to all types of SSRI to be associated with an increased prevalence of right ventricular outflow tract obstructions, which is an extension of the previously reported findings from Finland ${ }^{15}$ and consistent with findings from earlier studies from the USA. ${ }^{89}$ However, two recent cohort studies from the USA and the UK, respectively, suggested no substantial increase in the risk of cardiac birth defects associated with use of antidepressants during the first trimester. ${ }^{30} 31$ These findings are in accordance with our estimates when controlling for confounding by a sibling controlled design.

When we examined other specific birth defects reported in previous studies, we did not find an increased prevalence of hypospadias, limb reductions, gastroschisis, cystic kidneys, or craniosynostosis. One study $^{8}$ reported a fivefold increased prevalence of omphalocele associated with sertraline. We found a 


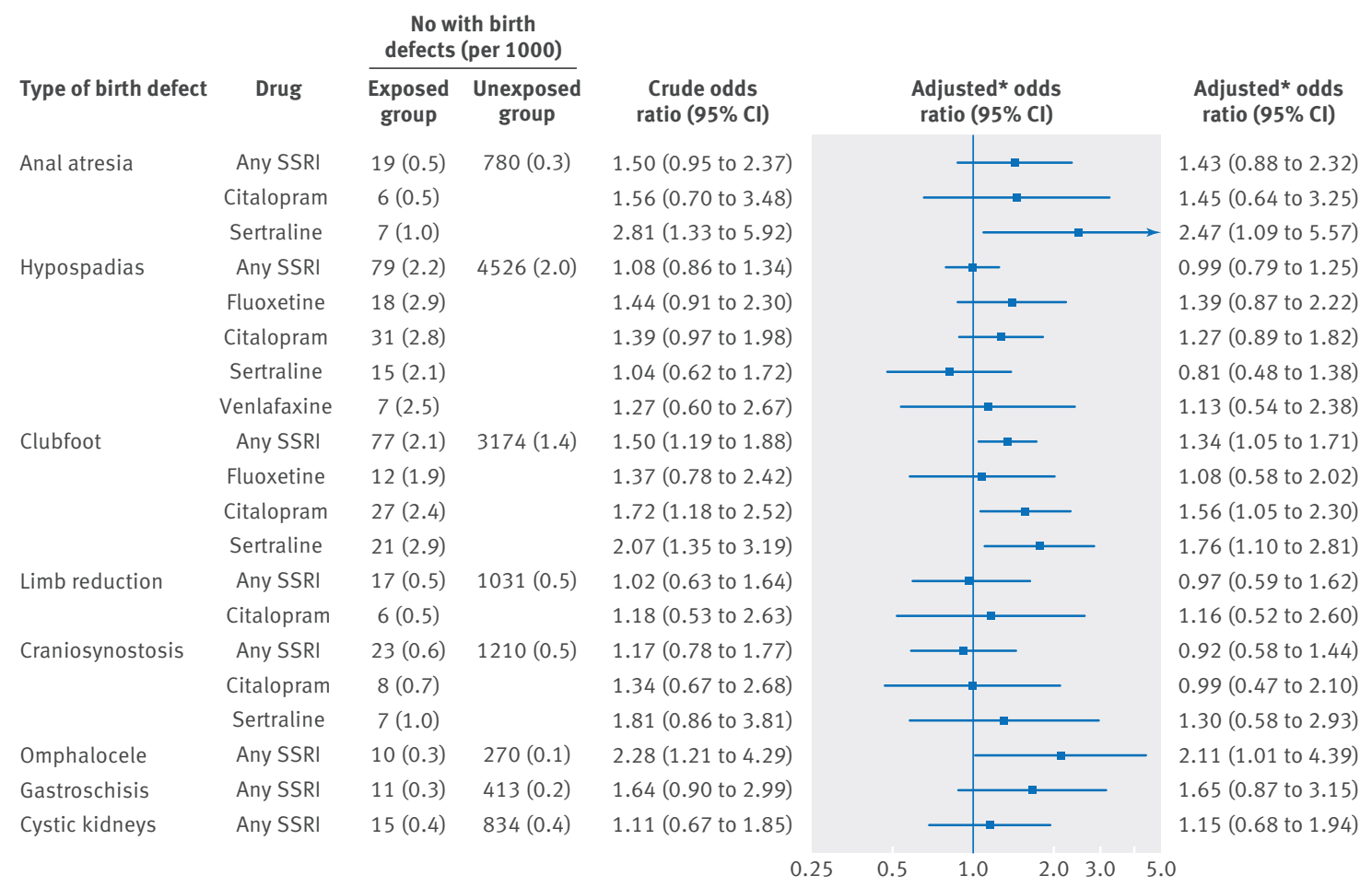

Fig 3 Selective serotonin reuptake inhibitors (SSRIs) or venlafaxine in early pregnancy and risk of non-cardiac birth defects. *Adjusted for maternal age, year of birth, birth order, smoking, maternal diabetes, and country, and use of other prescribed drugs (antiepileptics (ATC code N03), anxiolytics and hypnotics (N05B and N05C), and angiotensin converting enzyme inhibitors (C09))

twofold increased prevalence of omphalocele in infants exposed to SSRIs, but this estimate was based on only 10 cases. In our data, exposure to sertraline was associated with a 2.5-fold increased prevalence of anal atresia, corroborating but lower than the 3-4-fold increased odds previously estimated. ${ }^{8}$ With a prevalence of 0.5 per 1000 in SSRI exposed infants, the estimated absolute increase of anal atresia was low in our study.

Contrary to our results, a recent case-control study found positive associations between prenatal exposure to venlafaxine and various birth defects, including cardiac defects. ${ }^{21}$ The findings may, however, have been complicated by methodological limitations, including the small numbers included and a study design susceptible to recall bias.

Mechanism

Biological mechanisms remain unknown for most drug-defect associations, but a growing body of evidence from animal studies has suggested that the neurotransmitter serotonin is important as a signaling molecule in a variety of cells and tissues during embryogenesis, including heart development. ${ }^{32}$ Disruption of the serotonin signaling caused by exposure to SSRIs may thus result in disturbances of cardiac development, providing a biologically plausible explanation for the increased risk of cardiac birth defects. ${ }^{32-34}$

\section{Strengths and limitations of this study}

Our study was based on prospectively collected data from five Nordic countries, and the large numbers allowed us to study individual SSRIs and specific birth defects with independent ascertainment of exposure and outcome up to 1 year of age without any risk of recall bias. None the less, non-adherence to the dispensed antidepressants may have yielded misclassification of exposure and thus biased the relative estimates towards the null. However, previous Nordic studies comparing

\begin{tabular}{l}
$\begin{array}{l}\text { Table } 3 \text { | Selective serotonin reuptake inhibitors or venlafaxine in early pregnancy and birth defects. Odds ratios in unmatched and sibling controlled } \\
\text { analyses }\end{array}$ \\
\cline { 2 - 3 }
\end{tabular}


data on use of SSRIs from prescription databases with self reported SSRI use in pregnant women indicate the prescription data to be quite accurate and reliable for regular use of drugs such as antidepressants. ${ }^{35-37}$

Although we had data on several important confounders, including maternal age, diabetes, use of other potentially teratogenic drugs, and self reported smoking, residual confounding cannot be ruled out. By using dispensed drugs as a proxy for diabetes we may have missed those women with untreated diabetes type 2 . A Danish cross sectional study based on a population survey found that women with an unhealthy lifestyle were about 1.5-fold more likely to be current users of SSRIs than those with a healthy lifestyle. ${ }^{38}$ We were unable to include information on maternal use of alcohol or other lifestyle factors in our study. These factors may, however, be correlated with smoking. Thus adjusting for smoking may at least partly have controlled for some of the effect. Still, we find it likely that our estimates would have been even closer to 1.0 if we had been able to better control for lifestyle factors. This is further corroborated by our findings in the sibling controlled design. To use a sibling design when both exposure and outcome are rare, a large study population is required. Even though our study included 2.3 million births, the number in the sibling cohort was moderate. The sibling design has the advantage of controlling for familial factors that may be difficult to measure and control for in more conventional analyses. But sibling analyses reduce the size of the study population, since only sibling pairs discordant for both exposure and outcome are informative and contribute to the effect estimate. These births may represent a selected part of the population. Moreover, the sibling analysis has lower statistical power than the conventional cohort study design. Yet the upper limits of the confidence intervals were similar in the sibling analyses and the covariate adjusted cohort analyses. A possible weakness of the sibling design could be that use of SSRIs in a later pregnancy is not independent of the outcome of a first exposed pregnancy. However, in this study the order of exposure and outcome between discordant siblings did not influence the level of the odds ratios, which would suggest no such bias.

We tested several associations, but all the analyses were based on a priori stated hypotheses, which were built on previously published findings. As the number of tests in a study increases, the probability that one of them will be falsely positive also increases. We did not, however, adjust for multiple testing as this may increase the risk of type 2 errors. ${ }^{39}$ Considering lower statistical precision in the sibling controlled analyses, we focused on the major outcomes in these analyses, such as any birth defect, any cardiac defect, and right ventricular outflow tract obstruction defects.

Disentangling the potential teratogenicity of SSRIs from a potential effect of the underlying depression remains a challenge. We lacked information on severity of depression. We presumed, however, that women who had a previous hospital admission for a psychiatric disorder had more severe depression than women with no such admission. Restricting the analyses to women without previous hospital contacts for psychiatric disorders did not substantially change our relative estimates. Yet severity of the underlying illness may still vary substantially within this group of women.

We used population based data on live births, but we lacked information about prenatal diagnosis resulting in termination of pregnancy, miscarriages, or stillbirths caused by birth defects. If use of SSRIs is related to any of these outcomes, selection bias could be present. This may have led us to underestimate the association between use of SSRIs and birth defects. However, in an earlier study based on the same registers, no significant associations were found between use of SSRIs during pregnancy and risk of stillbirth or infant mortality. ${ }^{26}$ In addition there seems to be no association between use of antidepressants and late termination of pregnancy. ${ }^{40}$ Data on birth defects were obtained from medical registers, and previous validation studies suggest high validity of these diagnoses, with misclassification rates of $12 \%$ for both congenital malformations overall and cardiac defects. ${ }^{4142}$ We cannot, however, rule out that increased awareness of the potential for cardiac defects in infants exposed to SSRIs prenatally may have introduced surveillance bias and thereby inflated our relative estimates. Such bias would particularly concern less serious cardiac defects, such as ventricular septal defects, which often remain undiagnosed in infancy. We would, however, expect such differential misclassification to be of similar magnitude between the different types of SSRIs. Furthermore, the fact that we found no increased risk of cardiac defects in infants exposed to escitalopram argues against substantial surveillance bias. The risks of channeling bias are probably small considering similar pregnant populations and that prescription patterns of SSRIs to pregnant women differ somewhat between the Nordic countries-for example, citalopram and fluoxetine are the most prescribed SSRIs in Denmark, whereas citalopram and escitalopram are most prescribed in Norway. ${ }^{1243}$ We also have to consider that women who continue to use antidepressants in pregnancy may differ from those who were never prescribed antidepressants and those who stopped using antidepressants in pregnancy. Pregnancy is a major determinant of cessation of antidepressant treatment, which may be explained by concerns about potential adverse effects of the drugs. ${ }^{44}$

\section{Conclusions}

Taken together, the results from our covariate adjusted analyses and the sibling controlled analyses point against a substantial teratogenic effect of SSRIs. Our analyses did not suggest a teratogenic effect of venlafaxine either.

\section{AUTHOR AFFILIATIONS}

'Department of Pharmacoepidemiology, Division of Epidemiology, Norwegian Institute of Public Health, PB 4404 Nydalen, NO 0403 Oslo, Norway

2Department of Medicine Solna, Centre for Pharmacoepidemiology, Karolinska Institutet Stockholm, Sweden 
${ }^{3}$ Department of Global Public Health and Primary Care, University of Bergen, Bergen, Norway

4Department of Women's and Children's Health, Division of Obstetrics and Gynecology, Karolinska Institutet, Stockholm, Sweden

${ }^{5}$ Centre of Public Health Sciences, Faculty of Medicine, School of Health Sciences, University of Iceland, Reykjavik, Iceland ${ }^{6}$ Department of Epidemiology, Harvard T H Chan School of Public Health, Boston, MA, USA

${ }^{7}$ Department of Medical Genetics, University of Helsinki, Helsinki, Finland

${ }^{8}$ Department of Child Psychiatry, University of Turku, Turku, Finland ${ }^{9} \mathrm{THL}$ National Institute for Health and Welfare, Helsinki, Finland

${ }^{10}$ Nordic School of Public Health, Gothenburg, Sweden

${ }^{11}$ Teratology Information Service, HUSLAB and Helsinki University Central Hospital, Helsinki, Finland

${ }^{12}$ Department of Clinical Pharmacology, Helsinki University, Helsinki, Finland

${ }^{13}$ Department of Clinical Epidemiology, Institute of Clinical Medicine, Aarhus University Hospital, Aarhus, Denmark

We thank Gunnar Bergman (Department of Women's and Children's Health, Karolinska Institutet) for help with classifying the cardiovascular birth defects.

Contributors: $\mathrm{KF}, \mathrm{BH}, \mathrm{HK}$, and $\mathrm{MN}$ conceived and designed the study. $\mathrm{BH}, \mathrm{MG}, \mathrm{MA}, \mathrm{AE}, \mathrm{MN}$, and UAV acquired the data in their respective countries. KF and MN drafted the manuscript. HK obtained funding and supervised the study. BH was responsible for the statistical analyses. All authors participated in interpretation of data and critical revision of the manuscript. $\mathrm{KF}$ and $\mathrm{BH}$ had full access to all of the data in the study and take responsibility for the integrity of the data and the accuracy of the data analyses. They are the guarantors.

Funding: This study was funded by the authors' affiliations and the Swedish pharmacy company Apoteksbolaget. Apoteksbolaget had no role in the design and conduct of the study; the collection, management, analysis, and interpretation of the data; the preparation, review, or approval of the manuscript; or the decision to submit the manuscript for publication.

Competing interests: All authors have completed the ICMIE uniform disclosure form at www.icmje.org/coi_disclosure.pdf and declare: no support from any organisation for the submitted work; no financial relationships with any organisations that might have an interest in the submitted work in the previous three years; no other relationships or activities that could appear to have influenced the submitted work. Ethical approval: This study was approved by the regional ethical review board at Karolinska Institutet in Stockholm, Sweden (No 2008/1371-31/4); the Danish Data Protection Agency, Denmark; the National Institute for Health and Welfare (THL), the Social Insurance Institution of Finland and Statistics Finland, Finland; the National Bioethics Committee and the Data Protection Authority in Iceland, Iceland; and the Norwegian Data Inspectorate, Norway.

Data sharing: No additional data available

Transparency: The lead authors (KF and MN) affirm that the manuscript is an honest, accurate, and transparent account of the study being reported; that no important aspects of the study have been omitted; and that any discrepancies from the study as planned (and, if relevant, registered) have been explained.

This is an Open Access article distributed in accordance with the Creative Commons Attribution Non Commercial (CC BY-NC 4.0) license, which permits others to distribute, remix, adapt, build upon this work non-commercially, and license their derivative works on different terms, provided the original work is properly cited and the use is non-commercial. See: http://creativecommons.org/licenses/ by-nc/4.0/.

1 O'Keane V, Marsh MS. Depression during pregnancy. BMJ 2007:334:1003-5.

2 Kieler $\mathrm{H}$. The Nordic health registers-an important source when evaluating the safety of antidepressants during pregnancy. Clin Epidemiol 2010;2:205-7.

3 El Marroun H, Jaddoe VW, Hudziak JJ, et al. Maternal use of selective serotonin reuptake inhibitors, fetal growth, and risk of adverse birth outcomes. Arch Gen Psychiatry 2012;69:706-14.

4 Cooper WO, Willy ME, Pont SI, et al. Increasing use of antidepressants in pregnancy. Am J Obstet Gynecol 2007;196:544-5.

5 Hayes RM, Wu P, Shelton RC, et al. Maternal antidepressant use and adverse outcomes: a cohort study of 228,876 pregnancies. Am J Obstet Gynecol 2012:207:49.
6 Huybrechts KF, Palmsten K, Mogun H, et al. National trends in antidepressant medication treatment among publicly insured pregnant women. Gen Hosp Psychiatry 2013:35:265-71.

7 Tuccori M, Montagnani S, Testi A, et al. Use of selective serotonin reuptake inhibitors during pregnancy and risk of major and cardiovascular malformations: an update. Postgrad Med 2010;122:49-65.

8 Louik C, Lin AE, Werler MM, et al. First-trimester use of selective serotonin-reuptake inhibitors and the risk of birth defects. N Eng / Med 2007:356:2675-83.

9 Alwan S, Reefhuis J, Rasmussen SA, et al. Use of selective serotonin-reuptake inhibitors in pregnancy and the risk of birth defects. N Engl / Med 2007;356:2684-92.

10 Diav-Citrin O, Shechtman S, Weinbaum D, et al. Paroxetine and fluoxetine in pregnancy: a prospective, multicentre, controlled observational study. BrJ Clin Pharmacol 2008;66:695-705.

11 Pedersen LH, Henriksen TB, Vestergaard M, et al. Selective serotonin reuptake inhibitors in pregnancy and congenital malformations: population based cohort study. BMJ 2009;339:b3569.

12 Kornum JB, Nielsen RB, Pedersen L, et al. Use of selective serotoninreuptake inhibitors during early pregnancy and risk of congenital malformations: updated analysis. Clin Epidemiol 2010;2:29-36.

13 Reis M, Kallen B. Delivery outcome after maternal use of antidepressant drugs in pregnancy: an update using Swedish data. Psychol Med 2010;40:1723-33.

14 Wurst KE, Poole C, Ephross SA, et al. First trimester paroxetine use and the prevalence of congenital, specifically cardiac, defects: a meta-analysis of epidemiological studies. Birth Defects Res A Clin Mol Teratol 2010;88:159-70.

15 Malm H, Artama M, Gissler M, et al. Selective serotonin reuptake inhibitors and risk for major congenital anomalies. Obstet Gynecol 2011;118:111-20.

16 Oberlander TF, Warburton W, Misri S, et al. Major congenital malformations following prenatal exposure to serotonin reuptake inhibitors and benzodiazepines using population-based health data. Birth Defects Res B Dev Reprod Toxicol 2008;83:68-76.

17 Kallen BA, Otterblad OP. Maternal use of selective serotonin re-uptake inhibitors in early pregnancy and infant congenital malformations. Birth Defects Res A Clin Mol Teratol 2007;79:301-8.

18 Margulis AV, Abou-Ali A, Strazzeri MM, et al. Use of selective serotonin reuptake inhibitors in pregnancy and cardiac malformations: a propensity-score matched cohort in CPRD. Pharmacoepidemiol Drug Saf 2013;22:942-51.

19 Vasilakis-Scaramozza C, Aschengrau A, Cabral H, et al. Antidepressant use during early pregnancy and the risk of congenital anomalies. Pharmacotherapy 2013;33:693-700.

20 Connolly KR, Thase ME. If at first you don't succeed: a review of the evidence for antidepressant augmentation, combination and switching strategies. Drugs 2011;71:43-64.

21 Polen KN, Rasmussen SA, Riehle-Colarusso T, et al. Association between reported venlafaxine use in early pregnancy and birth defects, national birth defects prevention study, 1997-2007. Birth Defects Res A Clin Mol Teratol 2013;97:28-35.

22 Mitchell AA. Studies of drug-induced birth defects. In: Strom BL, Kimmel SE, Hennessy S, eds. Pharmacoepidemiology. Fifth edn. Wiley, 2012;487-504.

23 Furu K, Wettermark B, Andersen M, et al. The Nordic countries as a cohort for pharmacoepidemiological research. Basic Clin Pharmacol Toxicol 2010;106:86-94.

24 WHO Collaborating Centre for Drug Statistics Methodology. Guidelines for ATC classification and DDD assignment 2009. Norwegian Institute of Public Health, Oslo.

25 Kieler H, Artama M, Engeland A, et al. Selective serotonin reuptake inhibitors during pregnancy and risk of persistent pulmonary hypertension in the newborn: population based cohort study from the five Nordic countries. BMJ 2012;344:d8012.

26 Stephansson O, Kieler H, Haglund B, et al. Selective serotonin reuptake inhibitors during pregnancy and risk of stillbirth and infant mortality. JAMA 2013;309:48-54

27 Wogelius P, Norgaard M, Gislum M, et al. Maternal use of selective serotonin reuptake inhibitors and risk of congenital malformations Epidemiology 2006;17:701-4.

28 Reis M, Kallen B. Combined use of selective serotonin reuptake inhibitors and sedatives/hypnotics during pregnancy: risk of relatively severe congenital malformations or cardiac defects. A register study. BMJ Open 2013;3(2)

29 Bakker MK, Kerstjens-Frederikse WS, Buys CH, et al. First-trimester use of paroxetine and congenital heart defects: a population-based case-control study. Birth Defects Res A Clin Mol Teratol 2010;88:94-100

30 Huybrechts KF, Palmsten K, Avorn J, et al. Antidepressant use in pregnancy and the risk of cardiac defects. N Engl J Med 2014;370:2397-407.

31 Ban L, Gibson J, West J, et al. Maternal depression, antidepressant prescriptions, and congenital anomaly risk in offspring: a populationbased cohort study. BJOG 2014:121:1471-81. 
32 Sadler TW. Selective serotonin reuptake inhibitors (SSRIs) and heart defects: potential mechanisms for the observed associations. Reprod Toxicol 2011:32:484-9.

33 Nebigil CG, Maroteaux L. Functional consequence of serotonin/5-HT2B receptor signaling in heart: role of mitochondria in transition between hypertrophy and heart failure? Circulation 2003;108:902-8

34 Sari Y, Zhou FC. Serotonin and its transporter on proliferation of fetal heart cells. Int J Dev Neurosci 2003;21:417-24.

35 Olesen C, Sondergaard C, Thrane N, et al. Do pregnant women report use of dispensed medications? Epidemiology 2001;12:497-501.

36 Skurtveit S, Selmer R, Tverdal A, et al. Drug exposure: inclusion of dispensed drugs before pregnancy may lead to underestimation of risk associations. / Clin Epidemiol 2013;66:964-72.

37 Stephansson O, Granath F, Svensson T, et al. Drug use during pregnancy in Sweden-assessed by the Prescribed Drug Register and the Medical Birth Register. Clin Epidemiol 2011:3:43-50.

38 Laugesen K, Telen Andersen AB, Norgaard M, et al. Use of selective serotonin reuptake inhibitors and lifestyle among women of childbearing age: a Danish cross-sectional survey. BMJ Open 2013;3(7).
39 Rothman KJ. Six persistent research misconceptions. J Gen Intern Med 2014;29:1060-4

40 Kieler H, Malm H, Artama M, et al. Use of antidepressants and association with elective termination of pregnancy: population based case-control study. BJOG 2014, published online 14 Nov. doi:10.1111/1471-0528.13164.

41 Knudsen LB, Olsen J. The Danish Medical Birth Registry. Dan Med Bull 1998;45:320-3.

42 Jepsen P, Jepsen B, Johnsen SP, et al. Validity of diagnoses of cardiac malformations in a Danish population-based hospital-discharge registry. Int J Risk Safety Med 2006;18:77-81.

43 Nordeng H, van Gelder MM, Spigset O, et al. Pregnancy outcome after exposure to antidepressants and the role of maternal depression: results from the Norwegian Mother and Child Cohort Study. J Clin Psychopharmacol 2012;32:186-94

44 Petersen I, Gilbert RE, Evans SJ, et al. Pregnancy as a major determinant for discontinuation of antidepressants: an analysis of data from The Health Improvement Network. J Clin Psychiatry 2011;72:979-85

(C) BMJ Publishing Group Ltd 2015 